\title{
Weak collisionless shocks in laser-plasmas
}

\author{
R.A. Cairns ${ }^{1}$, R. Bingham ${ }^{2,3}$, R.G.M. Trines ${ }^{2}$ and P. Norreys ${ }^{2,4}$ \\ 1. School of Mathematics and Statistics, University of St \\ Andrews, St Andrews, KY16 9SS, UK. \\ 2. STFC Rutherford Appleton Laboratory, Chilton, \\ Didcot, Oxon. OX11 0QX, UK. \\ 3. Department of Physics, University of Strathclyde, Glasgow, \\ G4 0NG, UK. \\ 4. Department of Physics, University of Oxford, Oxford, \\ OX1 2JD, UK.
}

\begin{abstract}
We obtain a theory describing laminar shock-like structures in a collisionless plasma and examine the parameter limits, in terms of the ion sound Mach number and the electron/ion temperature ratio, within which these structures exist. The essential feature is the inclusion of finite ion temperature with the result that some ions are reflected from a potential ramp. This destroys the symmetry between upstream and downstream regions that would otherwise give the well-known ion solitary wave solution. We have shown earlier [Cairns et al, Phys. Plasmas 21, 022112 (2014)] that such structures may be relevant to problems such as the existence of strong, localized electric fields observed in laser compressed pellets and laser acceleration of ions. Here we present results on the way in which these structures may produce species separation in fusion targets and suggest that it may be possible to use shock ion acceleration for fast ignition.
\end{abstract}

\section{Introduction}

We have recently developed a simple analytic theory of weak collisionless shocks in an unmagnetized collisionless plasma [1] and argued that it may be relevant to observations of localized high electric fields in laser compressed pellets $[2,3,4,5]$ and also to production of ion beams with low energy spread [6]. In this paper we begin with a summary of the basic theory, extending our earlier work by including some results on the scaling of the key quantities and discussing in more detail the way in which the structures depend on the parameters of the problem. We then discuss its possible relevance to species separation in fusion targets to fast ignition using shock generated ion beams. 


\section{Theory}

We look for nonlinear structures that are stationary in an appropriately chosen frame of reference within a collisionless plasma. The theory is very similar to the well-known theory of ion sound solitary waves, except that we include a finite ion temperature. The result is that some of the incoming ions are reflected, so that the solitary wave becomes an asymetrical structure more like a shock.

We begin by assuming that in the rest frame of the structure the electric potential $\phi$ increases monotonically from zero far upstream to a maximum $\phi_{\max }$. If, in this frame, the upstream ions have a thermal spread and a mean velocity $V$, then the ion density upstream of the potential maximum is

$$
\begin{aligned}
n_{i}\left(\phi, \phi_{\max }\right)= & \frac{1}{\sqrt{2 \pi}} \int_{0}^{\infty} \exp \left[-\frac{\left(\sqrt{v^{2}+2 \phi}-V\right)^{2}}{2}\right] d v \\
& +\frac{1}{\sqrt{2 \pi}} \int_{0}^{\sqrt{2\left(\phi_{\max }-\phi\right)}} \exp \left[-\frac{\left(\sqrt{v^{2}+2 \phi}-V\right)^{2}}{2}\right] d v
\end{aligned}
$$

where the velocity has been normalized to the ion thermal speed, the density to that of the incoming ion flow and the potential to $\frac{m_{i} V_{i}^{2}}{Z e}$ with $V_{i}$ the ion thermal velocity, $m_{i}$ the ion mass, $e$ the electron charge and $Z$ the ion charge number.

In the case of the electrons, we assume that they take up an equilibrium Boltzmann distribution, flowing to produce charge neutrality far upstream, so that

$$
n_{e}\left(\phi, \phi_{\max }\right)=Z n_{i}\left(0, \phi_{\max }\right) \exp \left(\frac{\phi}{T}\right),
$$

where $T$ is the ratio of the electron to ion temperature. We have introduced $\phi_{\max }$ as a variable in these densities since it cannot be chosen arbitrarily but must be self consistent with the dynamics of the system. The potential is given by Poisson's equation which, if we scale lengths to $\frac{V_{i}}{\omega_{p i}}$, with $\omega_{p i}$ the ion plasma fequency based on the density of the incoming flow at $\phi=0$, becomes

$$
\frac{d^{2} \phi}{d x^{2}}=n_{e}\left(\phi, \phi_{\max }\right)-n_{i}\left(\phi, \phi_{\max }\right)
$$

There are two parameters in the problem, $T$ and $V$, though it is useful to replace the latter with a Mach number $M$ defined in terms of the ion sound speed $\sqrt{\frac{T_{e}}{m_{i}}}$, so that in our normalized variables $M=\frac{V}{\sqrt{T}}$. This is not the true Mach number since it neglects ion pressure, but is convenient and, since the electron temperature needs to be substantially above the ion temperature for the structures to exist, never very far from the true Mach number. As discussed 
in our previous paper [1], a self consistent solution for the upstream density ramp can only be found within a limited range of these parameters and where it does exist the solution downstream is typically a density oscillation, as illustrated in Figure 1. The asymmetry between the upstream and downstream behaviour arises from the presence of reflected ions upstream and only ions which cross the potential barrier, with a density given by the first term of Equation (1), downstream.

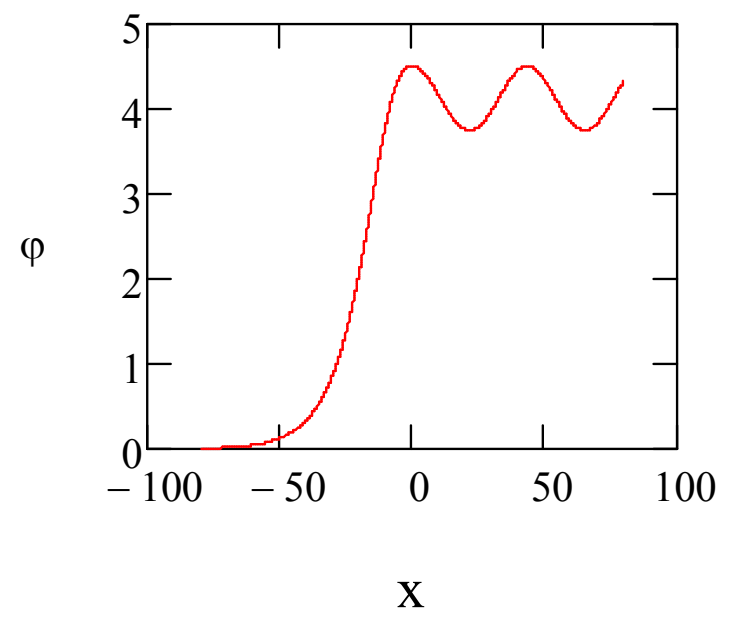

Figure 1. Normalized potential profile for $T=20, M=1.2$.

By numerical trial and error we have estimated the upper and lower Mach number limits within which solutions exist for a range of temperatures, with the result shown in Figure 2.

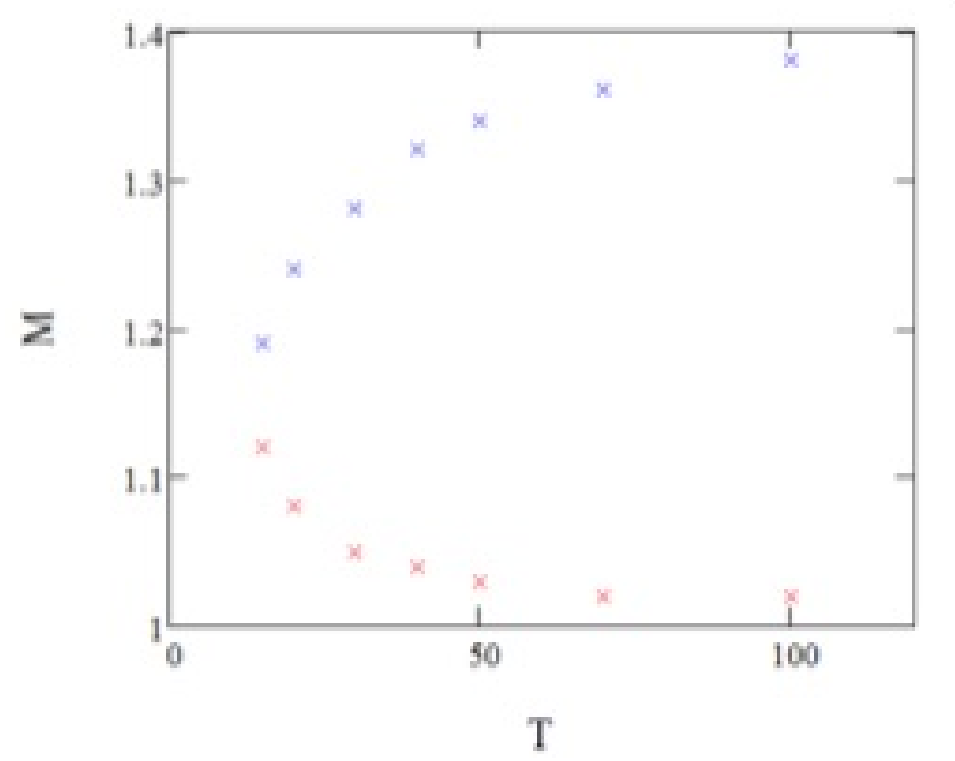

Figure 2. Upper (blue) and lower (red) limits of the allowed Mach number range, estimated by numerical trial and error for a range of electron/ion temperature ratios. 
There is a minimum electron/ion temperature ratio of a little below 15, with the range of allowed Mach numbers broadening above this value. The existence of a fairly low upper Mach number for these structures is consistent with early computer simulations carried out by Forslund and Freidberg [8], who found structures with the type of oscillatory behaviour we have found, involving a small number of reflected ions, up to a critical Mach number above which most ions were reflected and there was a population of trapped ions downstream. The requirement for a high temperature ratio can be understood from Poisson's equation. The potential ramp we assume needs $n_{e}>n_{i}$ when $\phi$ is small then $n_{e}<n_{i}$ for larger $\phi$, so as to give the correct curvatures. If $T$ is too small $n_{e}$ increases too rapidly with $\phi$ for the latter condition to be possible. As $T$ becomes large the lower Mach number limit approaches one, as might be expected. The existence of an upper Mach number limit is again connected with the need for a particular solution of Poisson's equation to exist. As the upper Mach number is approached the amplitude of the downstream oscillations decreases until very close to it there is essentially a constant potential downstream with $n_{e}=n_{i}$. Beyond this point the ions are slowed down too much and it becomes impossible to obtain a solution of the required form. As pointed out by Forslund and Freidberg the nature of the shock changes and we have not found any simple description of this. It is a problem we hope to address in the future, probably requiring the aid of simulations. Some recent simulations have been carried out by Macchi et al [9], investigating the time dependence of these structures as energy is transferred from the field to the accelerated ions. Consideration of this time dependence is beyond the scope of our simple model which may be expected to be valid over suitably short time scales.

For any given temperature ratio the fraction of ions reflected goes up with $M$. This is illustrated in Figure 2, together with the variation in $\phi_{\max }$, also increasing with $M$.

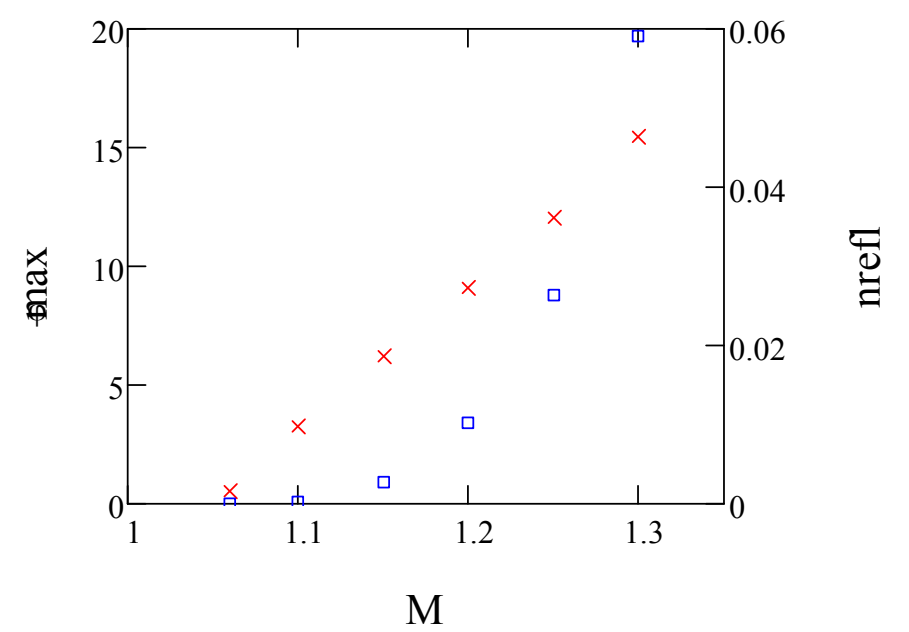

Figure 3. The normalized potential maximum (red crosses) and the reflected fraction (blue squares) for $T=30$. 
From Equation (1) it can be seen that the fraction of ions reflected is related to the other parameters by

$$
n_{r e f}=N\left(\sqrt{2 \phi_{\max }}-M \sqrt{T}\right)
$$

where $N$ is the cumulative normal distribution function. This is simply the value of the second term in the equation when $\phi=0$. In Figure 4 we show the dependence of this fraction on $T$ at the maximum allowed Mach number, the indication being that it levels off at a comparatively low value.

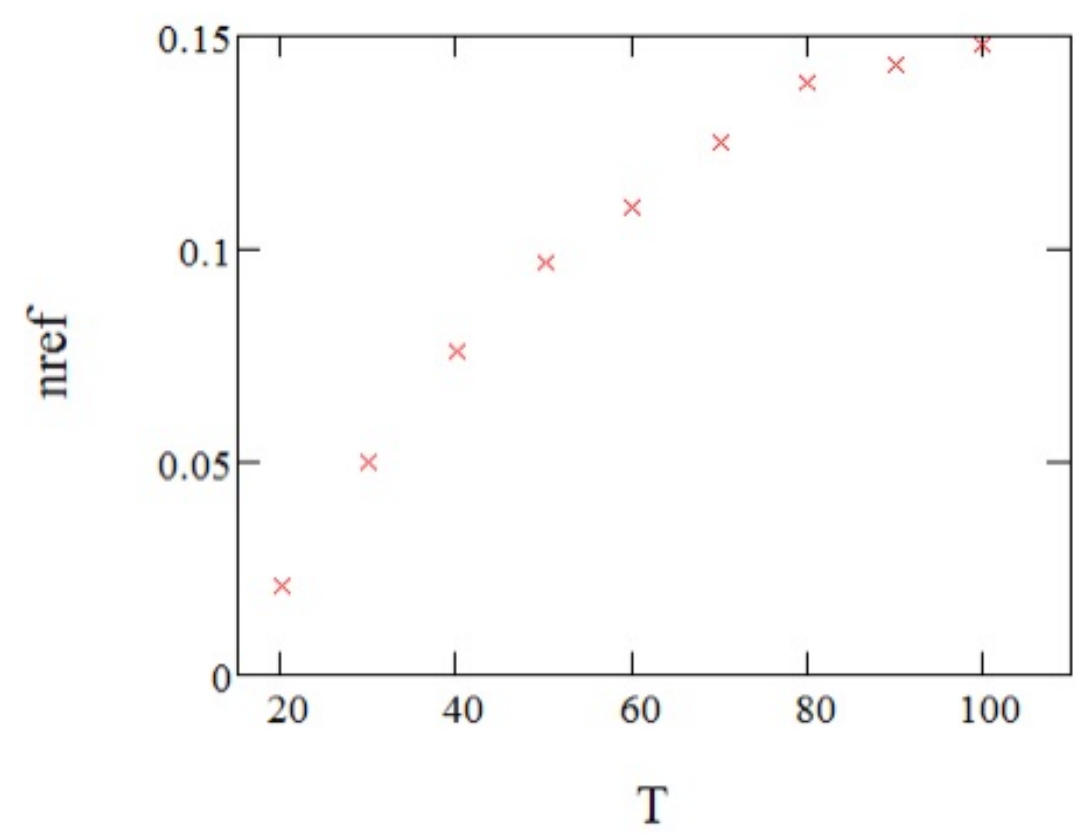

Figure 4. The reflected ion density as a function of temperature at the maximum Mach number.

Figure 5 shows the normalized potential maximum as a function of $T$, again at the maximum possible value of $M$, this time giving what appears to be a linear dependence. 


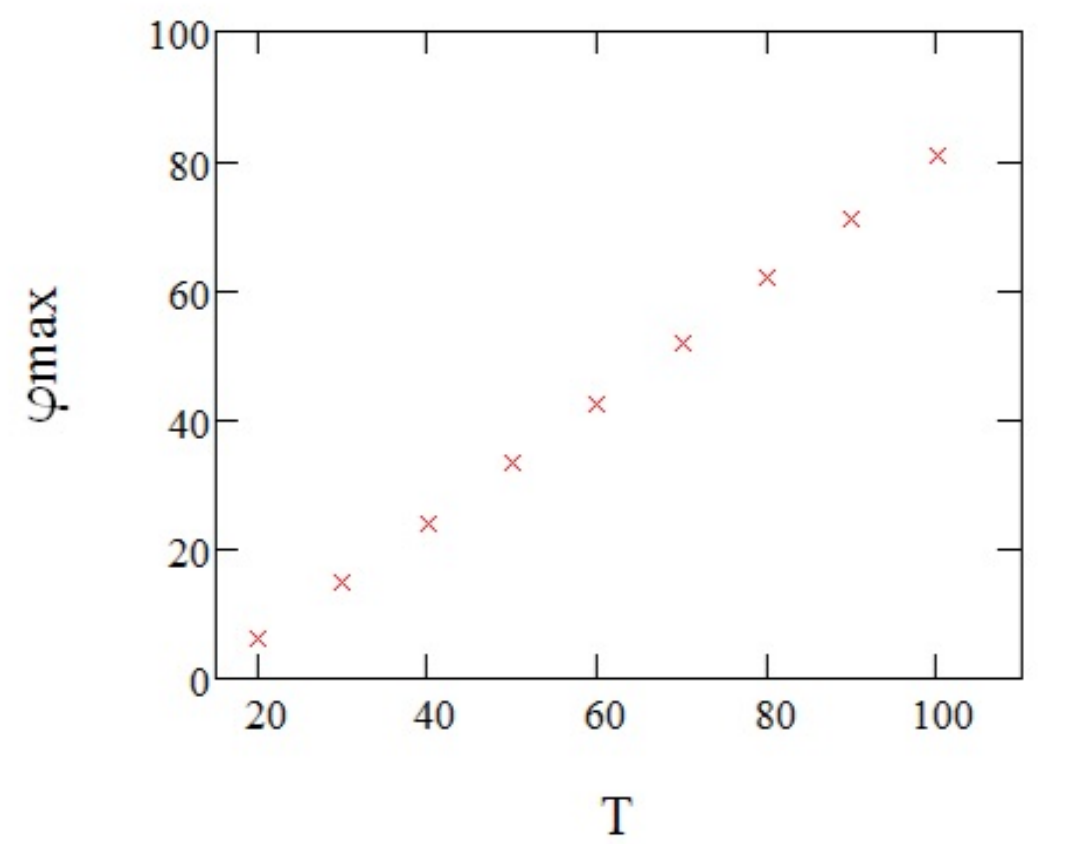

Figure 5. The normalized potential maximum as a function of $T$ at the highest allowed vaue of $M$.

The slightly irregular nature of these graphs is the result of the maximum allowed Mach number being estimated by a process of trial and error.

If we want to compare with experiment we need, of course, actual physical values of quantities rather than normalized values. We conclude this section by giving the conversions for electric field and length scale, namely

$$
\begin{aligned}
E(V / m) & =4.26 \times 10^{-3} E_{\text {norm }} T_{i}(k e V)^{1 / 2} n_{i}\left(m^{-3}\right)^{1 / 2} \\
L(m) & =2.35 \times 10^{5} L_{n o r m} T_{i}(k e V)^{1 / 2} n_{i}\left(m^{-3}\right)^{-1 / 2} Z^{-1} .
\end{aligned}
$$

The square root scaling with the plasma parameters has the consequence that the transformation to physical values is not too critically dependent on having the exact values of these parameters. Note also that the ion mass does not appear so the scaling is independent of the ion species. As we have shown previously, this type of structure appears to provide a plausible explanation for observations of high, localized electric fields in laser compressed pellets $[2,3$, $4,5]$ as an alternative possibility to the hydrodynamic shock with dissipation provided by pressure gradient driven diffusion suggested earlier [5]. We might also add that we obtain good agreement with experiments carried out more that 40 years ago by Taylor et al [10], in a regime many orders of magnitude away from laser compressed pellets.

We also showed previously that a weak laminar shock structure provides a good explanation for observations of ion acceleration in experiments of Haberberger et al $[6,11]$. The narrow spectrum with a sharp edge on the high energy side seen in these experiments is consistent with reflection of a small proportion of the ions, the shock front cutting off and reflecting only part of the incoming ion distribution. A higher Mach number shock, above the limit we have discussed above, would be expected to reflect a large fraction of the ions producing 
a much broader spectrum. This is possibly what is seen in other experiments on ion acceleration [12] showing a broader spectrum with a more symmetrical profile. If the shock Mach number can be controlled we suggest that it may be possible to obtain different ion acceleration regimes. A low Mach number shock can slice off a small part of the distribution and give a narrow spectrum with a sharp edge on the high energy side, while at higher Mach number most of the ions are reflected and a broader, more symmetrical energy spectrum generated. There is, of course, the disadvantage of the sharp spectrum in that it involves only a small fraction of the ions. Nevertheless, controlling the Mach number could lead to the ability to tailor a pulse to requirements to some extent.

\section{Species separation}

If the type of structure with localized high electric fields, as described in the previous section, arises spontaneously in compressed pellets then it could have implications for the separation of species in a deuterium-tritium target. The theory we have outlined can easily be extended to more than one ion species by adding extra terms to the right hand side of Equation(1). The normalization must, of course, be done with respect to one species and appropriate adjustments made to the expressions for any additional species. If for example we consider a plasma in which the incoming ions comprise a fraction $\lambda$ deuterium and $1-\lambda$ tritium then the density is given by $\lambda$ times the right hand side of (1) plus $1-\lambda$ times a similar expression with the potentials multiplied by $\frac{2}{3}$ to take account of the mass difference, the 2 in the denominator of the exponential replaced with $\frac{4}{3}$ and the whole thing multiplied by $\sqrt{\frac{3}{2}}$ to keep the normalization correct. The precise bounds for existence of the shocks and the other scalings discussed earlier are somewhat modified but the overall behaviour is, as would be expected, very similar. In Figure 6 we show the densities of deuterium and tritium in a shock with $T=20, M=1.2$, assumimg that the incoming flow comprises equal densities of the two species.

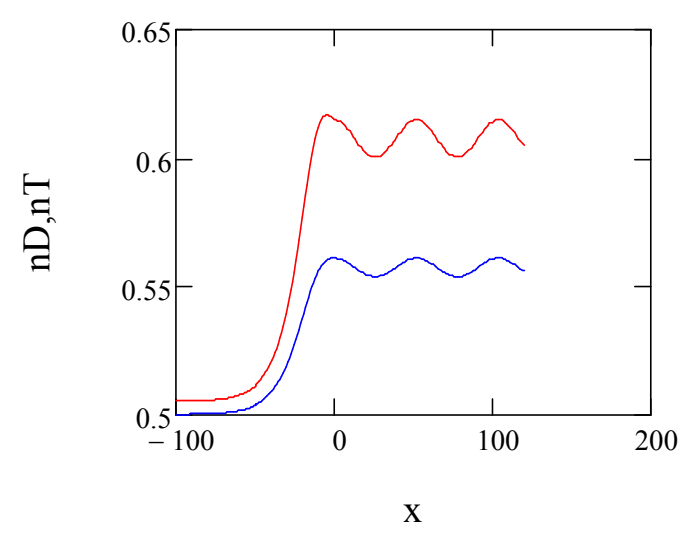

Figure 6. Normalized deuterium (red, upper curve) and tritium (blue, lower curve) densities for $T=20, M=1.2$. 
There is a small density difference upstream, resulting from the reflection ot the heavier tritium ions being very small, but a bigger difference downstream because of the greater slowing down of the deuterium ions. Recent work by Bellei and Amendt [13] also considers species separation in shocks and its possible impact on fusion rates, but differs from our work in that they consider much thicker hydrodynamic shocks.

\section{$4 \quad$ Fast ignition with shock accelerated ions}

Fast ions have been proposed as a way of producing fast ignition in fusion targets, the usual idea being that ions would be generated by a laser beam separate from that doing the pellet compression with a separate target as described in a recent review by Fernandez et al [14]. More recently it has been suggested by Naumova et al that fast ions driven by the ponderomotive force of a very intense tightly focused laser pulse might be used [15]. This scheme involves a pulse of intensity in excess of $10^{22} \mathrm{~W} / \mathrm{cm}^{2}$ focused so as to bore a hole in the compressed pellet. Since ion acceleration can be achieved with shocks, we suggest that it may be possible to launch a shock around the critical surface in order to accelerate ions inwards towards the compressed core. Since no hole boring is involved it should be possible in principle to do this in a spherically symmetrical way. In a stationary plasma the reflected ion velocity is around twice the shock speed and, if a shock of Mach number $M$ is excited, this gives a speed $2 M \sqrt{\frac{T_{e}}{m_{i}}}$ and hence an ion energy of $2 M^{2} T_{e}$. For this purpose we would propose generating a shock with a Mach number above the critical value we discussed earlier, since this then pushes most of the ions in front of it and a very narrow energy spectrum seems unnecessary for this purpose.

A crucial question is of course whether ions accelerated in this way can penetrate to the core of the pellet. To investigate this we have used the ion slowing formula given by Li and Petrasso [16], looking at penetration of hydrogen ions into a dense plasma with equal deuterim/tritium concentrations at a temperature of $10 \mathrm{keV}$ and the density profile shown in Figure 7. For the Coulomb logarithms we have taken 3 for ion-electron collisions and 10 for ion-ion collisions, approximating the values given by $\mathrm{Li}$ and Petrasso. The energy loss as a function of distance is shown in Figure 7. 


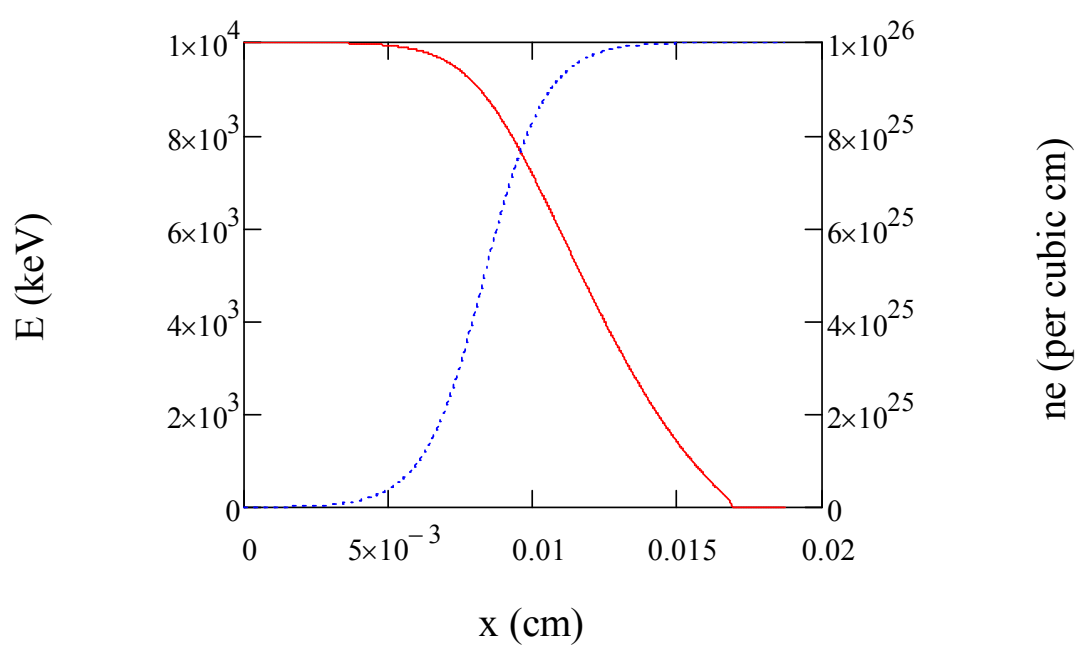

Figure 7. Energy (full red line) as a function of distance for a $10 \mathrm{MeV}$ particle going into a $10 \mathrm{keV}$ plasma with the density profile given by the blue dotted line.

In Figure 8 we show the corresponding energy deposition profile.

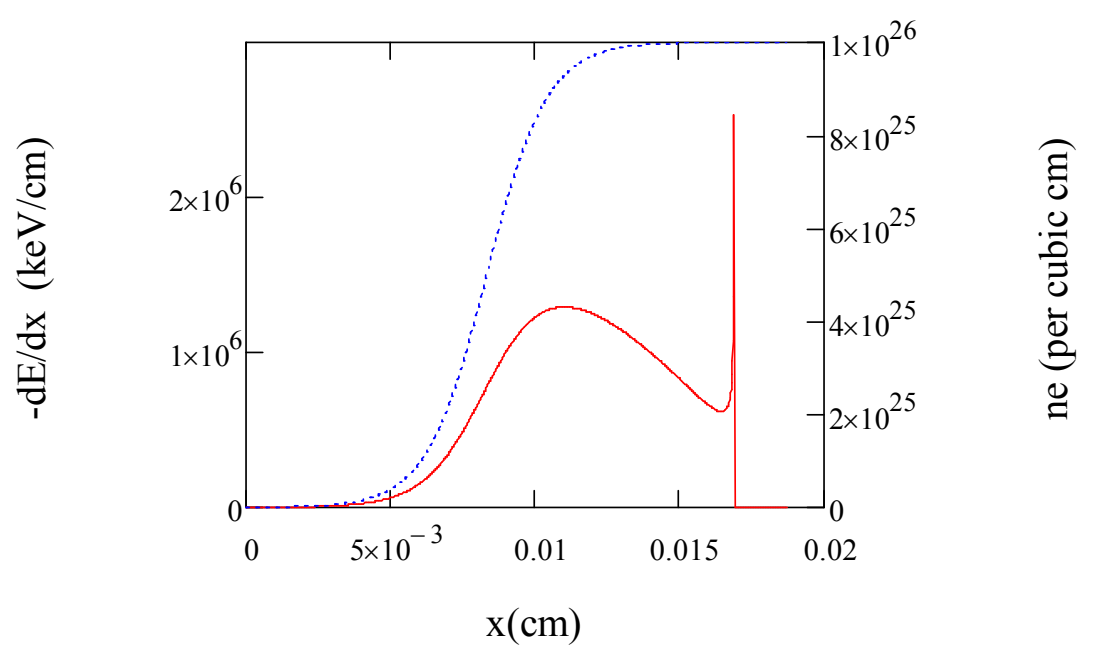

Figure 8. The energy loss rate corresponding to Figure 7.

Note that for this initial energy there is very little energy loss until the density reaches a value of around $10^{25} \mathrm{~cm}^{-3}$. The sharp cut off of the absorption is the result of the inclusion of a term with a Heaviside function in the $\mathrm{Li}$ and Petrasso formula. While there is a sharp peak in the energy absorption rate at the end of the ion trajectory most energy is lost in the earlier broad peak.It seems then that ions of around $10 \mathrm{MeV}$ energy which, according to our above estimates could be produced by a shock of Mach number around 2 in a plasma with electron temperature of the order of $1 \mathrm{MeV}$, would be capable of penetrating to the pellet core. If we wished to deposit around $20 \mathrm{KJ}$ of energy in the core then the number of $10 \mathrm{MeV}$ ions needed would be of the order of $10^{16}$.

In contrast to schemes of shock ignition involving compression of the core by a hydrodynamic shock [17] we are suggesting heating of the core by ions 
accelerated by a collisionless electrostatic shock in the outer region of the pellet. While many questions remain regarding the feasibilty of raising the temperature around the critical surface by the required amount, the generation of the shock and the efficiency with which it might accelerate ions, we suggest that the estimates we have given here show that this is an idea worth pursuing further and we propose to do so in future work.

\section{Conclusions}

In this paper we have given a brief summary of our earlier work weak collisionless shocks, extending the basic theory to give more details on how the shock structures depend on the parameters of the problem and attempting to explain the physical reasons why these structures only exist within a limited parameter range. We then outline the extension of the theory to more than one ion species and show that if such shocks arise in laser compressed deuterium-tritium targets they may produce significant charge separation, with possible consequences for fusion rates. Finally we discuss what we believe to be a novel proposal for fast ignition of targets by ions accelerated by a collisionless shock in the hot corona of a compressed target. Some initial estimates suggest that ions produced in this way could penetrate to the dense core and deposit their energy there.

\section{Acknowledgement}

This work was supported by the UK Science and Engineering Research Council

\section{References}

[1] R.A. Cairns, R. Bingham, P. Norreys and R. Trines, Phys. Plasmas, 21, 022112 (2014)

[2] C.K. Li, C.K Li, F.H. Seguin, J.R. Rygg, J.A. Frenje, M. Manuel, R.D. Petrasso, R. Betti, J. Delettrez, J.P. Knaur, F. Marshall, D.D.Meyerhofer, D. Shvarts, V.A. Smalyuk, C. Stoekl, O.L. Landen, R.P.J. Town, C.A. Back and J.D. Kilkenny, Phys. Rev.Lett. 100, 225001 (2008)

[3] J.R. Rygg, F.H. Seguin, C.K Li, J.A. Frenje, M.J.E Manuel, R.D. Petrasso, R. Betti, J. A. Delettrez, O.V. Gotchev, J.P. Knauer, D.D. Meyerhof, F.J. Marshall, C. Stoekl, and W. Theobald, Science 319, 1223 (2008).

[4] P.A. Amendt, J.L. Milovich, S.C. Wilks, C.K. Li, R.D. Petrasso, and F.H. Seguin, Plasma Phys. Controlled Fusion 51, 124048 (2009). 
[5] P.A. Amendt, S.C. Wilks, C. Bellei, C.K. Li and R.D. Petrasso, Phys. Plasmas 18, 056308 (2011).

[6] D. Haberberger, S. Tochitsky, F. Fi uza, C. Gong, R. Fonseca, L. O. Silva, W. B. Mori and C. Joshi, Nature Physics 8, 95 (2012).

[7] R. Sagdeev, in Reviews of Plasma Physics, edited by M.A. Leontovich (Consultants Bureau, New York, 1966), Vol. 4.

[8] D.W. Forslund and J.P. Freidberg, Phys. Rev. Lett. 27, 1189 (1971).

[9] A. Macchi, A.S. Nindrayog and F. Pegoraro, Phys. Rev. E 85, 046402 (2012).

[10] R.J. Taylor, D.R. Baker and H. Ikezi, Phys. Rev. Lett. 24, 206 (1970).

[11] F. Fiuza, A. Stockem, E. Boella, R. A. Fonseca, L. O. Silva, D. Haberberger, S. Tochitsky, C. Gong, W. B. Mori, and C. Joshi, Phys. Rev. Lett. 109, 215001 (2012).

[12] Z. Najmudin, C. A. J. Palmer, N. P. Dover, I. Pogorelsky, M. Babzien, A. E. Dangor, G. I. Dudnikova, P. S. Foster, J. S. Green, M. Ispiriyan, D. Neely, M. N. Polyanskiy, J. Schreiber, P. Shkolnikov, and V. Yakimenko, Phys. Plasmas 18, 056705 (2011).

[13] C.Bellei and P.A. Amendt, Phys. Rev. E 90, 013101 (2014)

[14] J.C. Fernandez, B.J. Albright, F.N. Beg, M.E. Foord, B.M. Hegelich, J.J. Honrubia, M. Roth, R.B. Stephens and L. Yin, Nucl. Fusion 54, 054006 (2014).

[15] N. Naumova, T. Schlegel, V. T. Tikhonchuk, C. Labaune, I.V. Solokov, and G. Mourou, Phys. Rev. Lett. 102, 025002 (2009).

[16] C.-K. Li and R. Petrasso, Phys. Rev. Lett. 70, 3059 (1993).

[17] R. Betti, C. D. Zhou, K. S. Anderson, L. J. Perkins, W. Theobald, and A. A. Solodov, Phys. Rev. Lett. 98, 155001 (2007). 